\title{
Ovarian and uterine tube infarction in a two years old toddler, case report
}

\begin{abstract}
Introduction: Sudden abdominal pain in toddlers is a taxing challenge, the only symptoms that a two years old toddler can present are crying in pain, it represent a great problem for young mother who never experienced such event before, as a little girl she can't complain verbally except crying and occasionally vomiting.

Case presentation: We present a case of a two years old girl suffered from severe abdominal pain associated with vomiting. She was referred to our clinic for surgical consultation, with three days history of abdominal pain, vomiting .Following her visit to the general practitioner, Full blood count revealed notable leukocytosis 24800 , with normal urinalysis, the abdomen was rigid, with guarding mainly in the right lower quadrant with absent bowel sounds, since the child was extremely agitated and un cooperating, abdomen X ray, Echo, CTor MRI were omitted. Despite its rarity we were in doubt of adnexal involvement. Through a $25 \mathrm{~mm}$, lower abdomen Rt sided skin crease Incision, the diagnosis of Rt sided Ovary and Uterine Tube Hemorrhagic infarction was confirmed clinically, the appendix appeared to have reactive inflammation. Resection of Rt Uterine Tube and Ovary, as well as appendectomy performed. Her post-operative course was uneventful. The Histology report confirmed the Diagnosis of Hemorrhagic Infarction of Rt Tube and Ovary, Appendix Reactive Inflammation and benign Lymphoid Hyperplasia.
\end{abstract}

Conclusion: Abdominal pain in toddler should be regarded as real emergency, and in females. Torsion of Ovary and Tube should always be kept in mind in the deferential diagnostic process.

Keywords: abdominal emergency, children, ovarian torsion, hemorrhagic infarction of tube and ovary, ovarian torsion, abdominal pain in female toddlers, pediatric emergency
Volume 4 Issue 5 - 2017

\author{
Muhammad Assem Kubtan \\ Head of Department of Surgery, Medical Faculty, Syrian Private \\ University, Syria
}

\begin{abstract}
Correspondence: Muhammad Assem Kubtan, Head of Department of Surgery, Medical Faculty, Syrian Private University, Syria, Tel 009-639-442-7I2-15,
\end{abstract} Email makubtan@gmail.com

Received: June 24, 2014 | Published: July 18, 2017
Abbreviations: US, ultra sound: CT, computerized tomography; MRI, magnetic resonance imaging; WCC, white cell count

\section{Introduction}

Abdominal pain in toddlers is a taxing challenge, ${ }^{1}$ a little child can only cry when in pain, may vomit and goes of food, obviously mother can notice the changes in her child but may be too late. Pain in right lower abdominal quadrant can direct physician towards a surgical emergency especially when other causes are ruled out by pediatric expert, Pediatric patients account only for about $15 \%$ of all cases of ovarian torsion 2 , in teenagers Ovarian torsion is one of the "cannot miss"diagnoses that presents with abdominal pain, and it must be considered in every case of abdominal pain in a female patient. ${ }^{2}$ When a definite diagnosis is made on clinical basis and investigative findings a decision of surgery should be taken speedily and promptly, and any delay might precipitate an unwanted results.

\section{Case presentation}

A two years old white girl referred to our surgical clinic with history described by mother as abdominal pain, crying for three days and vomiting, usually she is quite pleasant and healthy little girl, with normal delivery, and received all her vaccines. She was taken to her GP who asked for Full Blood Count and Urinalysis, the hematology report reviled high WCC at 24800, and Neutrophils $65 \%$, no abnormality in Urinalysis. On examination she was crying, extremely agitated and in pain, abdomen was rigid with clear guarding in the right lower abdomen, bowel sounds were absent. We admitted her to hospital and pediatric consultation ruled out any medically treatable cause, and agreed to the necessity of surgical exploration keeping in mind the remote possibility of acute appendicitis at this age, despite the importance of performing abdomen $\mathrm{X}$ ray, Echo and $\mathrm{Ct}$ we decline to perform them as the child was extremely agitated and un cooperating, as it was obvious that we are dealing with real surgical emergency, we decided to explore the abdomen through a skin crease incision in Rt lower quadrant, as if we are doing Appendectomy, bearing in mind the possibility of the need for larger incision in case of necessity. Through $25 \mathrm{~mm}$ Langs Lines skin incision the abdomen was opened, a very dark burgundy color and clotted blood recovered, with very dark red blackened lesion deep in noticed Figure 1, This lesion was gently delivered out and found to be consistent with right sided torsion of a $6 \mathrm{~cm}$ Uterine tube and engorged Ovary, the color was extremely dark red towards black swollen, edematous and compatible clinically with Hemorrhagic Infarction due to torsion of the Tube and Ovary Figure 2. The torsion was untwisted and despite the absence of any viable signs, a worm saline pack applied on clinically infarcted ovary for over 15 minutes with no viability of the tube and ovary recovered, a decision of resection was taken and both organs were resected Figure 3. Following we checked on the appendix, it was retro cecum and appeared to have reactive inflammation, it was also resected Figure 4, Wound closed with intra dermal suturing Figure 5. 


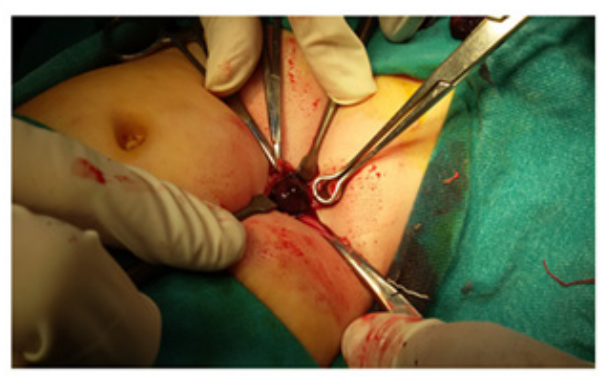

Figure I First appearance of infarcted ovary.

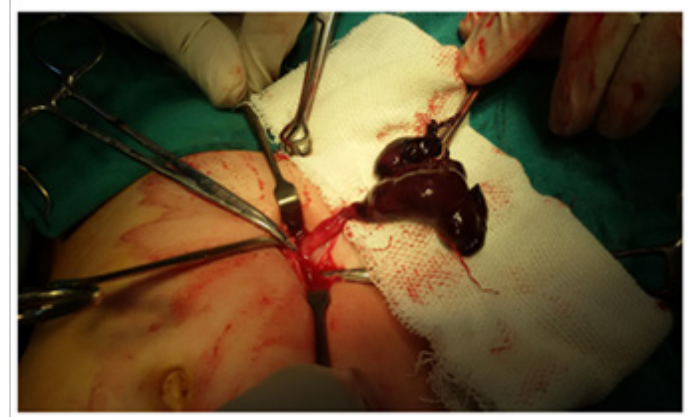

Figure 2 Infarcted ovary after de torsion and failure to restore viability.

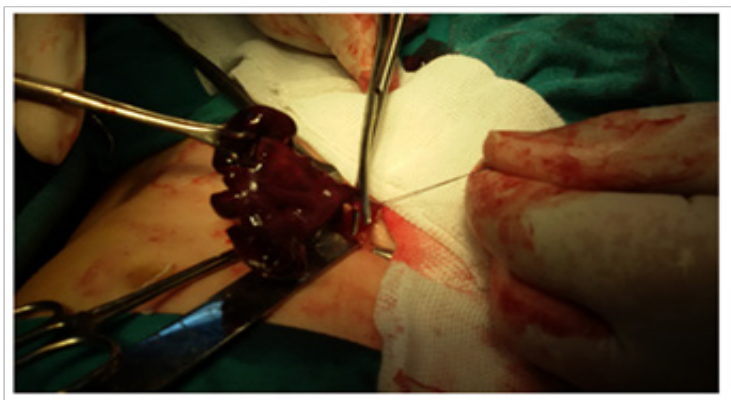

Figure 3 Resection of Rt ovary and uterine tube.

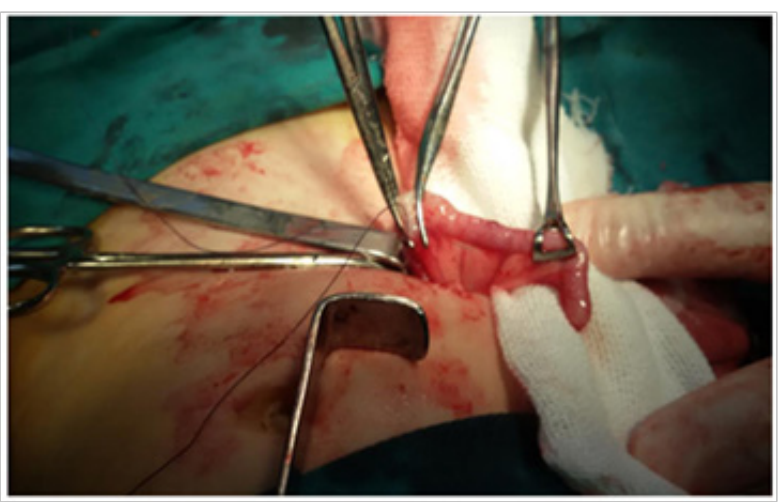

Figure 4 Reactive inflammation of appendix.

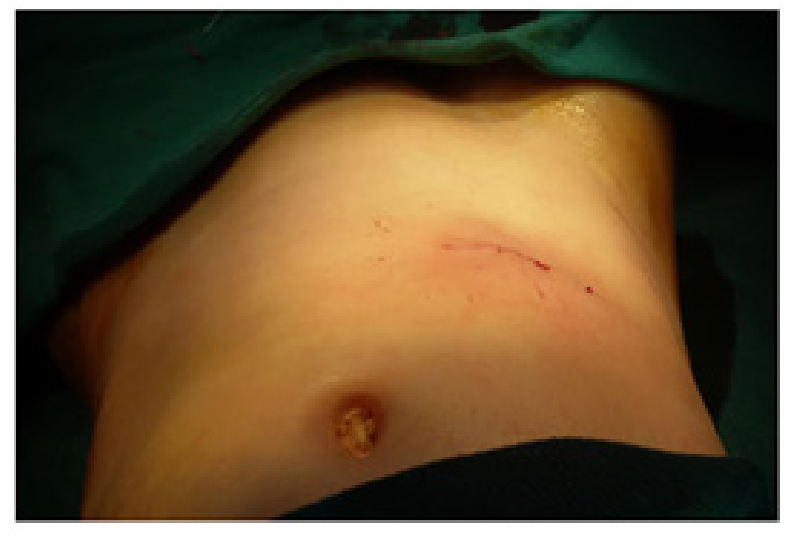

Figure $\mathbf{5}$ skin crease incision being closed subcutaneously.
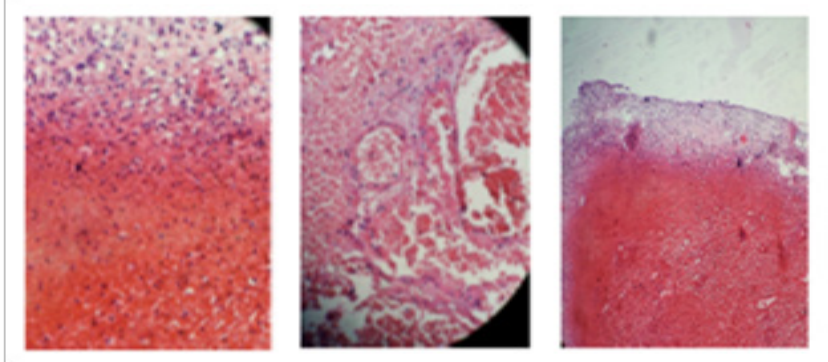

Figure 6 Hemorrhagic infarction of Rt ovarian tube and ovary.

\section{Discussion}

Despite all efforts made to solving Uterine Tube and Ovary, the time factor is extremely important to save Adnexal, and obviously three days delay a convincing evidence of the hopeless efforts Figure 6. Abdominal pain is a serious complaint for toddlers as their symptoms consist of just crying, obviously when mother has the experience she would be aware of the seriousness of such complaint and hurry to see a doctor, in children over 5-8 years of age, lower abdominal symptoms and following taking a proper history, reliable clinical examination and other investigations, the common deferential diagnosis include either Mesenteric Adenitis, or Acute Appendicitis etc. When in female toddler around the age of two years, abdominal symptoms should be regarded as more serious since almost always acute appendicitis can be ruled out, and involvement of adnexal should be kept in mind despite its rarity, ${ }^{3}$ It can occur at any age, for almost $70 \%$ of cases In women aged less than 20 years. ${ }^{4}$ Accordingly a quick and confident diagnosis is required to save the adnexal structures from infarction, this can't be achieved unless be thought of. Obviously it is always preferable to perform an abdomen X ray, abdominal US, Color Doppler 7, or CT or MRI of the abdomen ${ }^{5,6}$ those tools are extremely valuable and will usually confirm the diagnosis in older patients, but in our belief all this tests commonly require at least sedation or even anesthetizing the little girl, otherwise all those investigations without stabilizing the patient can't be relied on their results, it is very important to rely on the Surgeons experience and suspicion of the possibility of adnexal torsion. The question is raised on choosing the surgical approach whether open or laparoscopic intervention, and in case of open surgery should we use a skin crease or midline incision, we prefer 
the first choice and if we were faced with unsolvable finding problem we can always switch to other incision, Some surgeons advocate Laparoscopic distortion as a recognized and the mainstay of treatment regardless the condition of the ovaries. ${ }^{7,8}$ Further a question still be raised in case of infracted ovary and after failing in restoring viability of the organ and being convinced that it is infracted, we believe that it is waste of time to try to do opheropexy remains controversial and we prefer resection of the infracted ovary.

\section{Conclusion}

Adnexal torsion despite its low incidence should always be kept in mind when examining little girls around 2-3years old and even less, making early diagnosis and early surgical interference might save the Ovary, Uterine Tube and restore its viability, the use of diagnostic tools, such as Abdominal US, Ct, and MRI is extremely helpful in general and in cooperating patients but not always in little toddlers, the experience of Surgeon is very important in dealing with such cases, also early visit to physician is of paramount value.

\section{Acknowledgements}

I wish to extend my appreciation for the support provided by Professor N Ibrahim President of Syrian Private University, and Professor N Daher Deen of the Medical Faculty.

\section{Conflict of interest}

The author declares no conflict of interest.

\section{References}

1. Huang TY, Lau BH, Lin LW, et al. Ovarian cyst torsion in a toddler. Am J Emerg Med. 2009;27(5):632.

2. Eric R Schmitt, CA Marianne Gausche-Hill. Diagnosing and Managing Ovarian and Adnexal Torsion in Children. Los Angeles, USA: Harbor UCLA Medical Center; 2012.

3. Naveen Poonai, Caroline Poonai, Rodrick Lim, et al. Pediatric ovarian torsion: case series and review of the literature. Can J Surg. 2013;56(2):103-108

4. Vineet Mishra, ShaheenHokabaj, Priyankur Roy, et al. Case series of paediatric adnexal torsion: rare yet urgent entity. Case Report. 2017;6(1).

5. By Jennifer Eng-Lunt, Heather Appelbaum, Jahn Avarello. Pediatric and adolescent ovarian torsion; 2011.

6. Shauna Duigenan, Esther Olivan, Susanna I Lee. Varian torsion: diagnostic features on CT and MRI with Pathologic correlation. American Journal of Roentgenology. 2012;198:W122-131.

7. Abdul Ghani Nur Azurah, Zakaria Wan Zainol, Ani Amelia Zainuddin, Pei Shan Lim, et al. Update on the management of ovarian torsion in children and adolescents. World J Pediatr. 2015;11(1):25-40.

8. JH Ahn. Successful laparoscopic surgery for 14-month-old infant with ovarian. Obstet Gynecol Sci. 2014;57(2):160-163. 